\title{
Constant distillate composition of batch distillation column with variable reflux mode based on still pot concentration
}

\author{
Naseer A. Alhaboubi* \\ Chemical Engineering Dept., Al-Nahrain University, Baghdad 10071, Iraq
}

Received: 27 April 2021 / Accepted: 20 December 2021

\begin{abstract}
The objective of this work is to control the constant distillate composition of batch distillation column $x_{D}$. A new correlation developed involved the instant reflux ratio value $R$ with a composition of the remaining mixture in the bottom pot (boiler) $x_{B}$. Also adding the dynamic time as ahead time $(+d t)$ to the control algorithm to increase the $R$ to anticipate the changes in $x_{D} .+d t$ was calculated using an electroconductive tracer that was injected both in the bottom pot and in the top tray to estimate the upward and the downward dynamic time. The proposed correlation was successfully applied as a control strategy on a glass batch distillation pilot plant that has $5 \mathrm{~cm}$ diameter, $1 \mathrm{~m}$ long, eight sieve trays, and Methanol-Water mixture as a system. The real-time experimental runs of the suggested control algorithm applied by manipulating the reflux stream that affects the changes that happened in the top composition product to keep it constant. The proposed procedure shows a quick and stable response for distillate product composition during the operating time that minimizes and saves the energy supplied to the boiler. Moreover, the system gives a straight, smooth linear constant distillate product even in case of disturbing the system.
\end{abstract}

\section{Introduction}

Batch Distillation (BD) is one of the oldest separation units, historical development from the first applications more than 5000 years ago in Mesopotamia (today is known as Iraq) [1]. BD is one of the most important unit operations and the most widely used separation process in industrial separation chemicals used in small-scale production. BD is preferred to continuous distillation where small quantities of high-tech and high value-added chemicals and biochemicals can be separated. The batch unit requires less capital to separate relatively pure components. The most notable feature of BD is its versatility, so distillation is widely used in the pharmaceutical industry to purify and recover highvalue liquid components. It has better operational flexibility as well as reduces equipment costs when compared to continuous distillation [2, 3].

Generally, the distillation column is operated normally either in continuous or batch mode. The column is operated by the continuous method in a steady state after the startup period until it is closed. On the other hand, in batch mode, the column states change continuously over time resulting in non-linear and non-stationary [4].

Also, the advantage of BD has of being much more flexible than continuous distillation, as it has more degrees of freedom (e.g., temperature, flow rate, pressure). Flexibility allows for handling different feed compositions and product specifications; Also, a wide range of different mixtures can be separated using the same batch column. This is a big advantage with the ever-changing product specification requirements in the market today. Moreover, BD often means a simpler process and lower capital cost than continuous distillation [5].

In $\mathrm{BD}$, the researcher's concern for the last decades about: the design of alternative and suitable column configurations, the development of mathematical models, the formulation and solution of dynamic optimization problems for optimal design, control, and operation [6].

The use of BD despite the industry's competitiveness has increased over the past years, which makes the interest in process modelling and the use of this model to optimize and reduce energy requirements (which means, in practice, minimizing production time) and product loss, and always maintaining High purity requirements [7].

It is this flexibility and the inherent unsteady nature of the process which pose challenging design and operation problems. There are two methods of operating BD columns: variable reflux and constant reflux, resulting in constant product composition and variable product composition at distillate of the key component, respectively (Bogart [8] presented the first analysis of the variable reflux for a binary system).

\footnotetext{
* Corresponding author: naseer.a.alhaboubi@nahrainuniv.edu.iq
} 
Diwekar and Madhavan [6] added a third operating mode to the $\mathrm{BD}$, in addition to the previous two modes (fixed reflux rate with variable distillate composition, and variable reflux rate with fixed distillate composition) which is an optimal reflux rate with an optimal distillate composition. Defined the last operating mode like the one leading to the most profitable operation. Compared with other traditional operating modes, their optimal reflux rate was also a policy of increasing reflux policy led to the shortest batch time.

Sørensen [9] studied the cyclic operation and its useful execution in BD. It comprises of an alternation of three assignments: a reflux drum filling arranged underneath the condenser, complete distillate evacuation into a tank, and total reflux from the reflux drum with differing hold-up.

Also, built up a heuristic equation utilizing the gPROMS software for tracking down the total number of cycles to contemplate the cyclic working policy for a $\mathrm{BD}$ column arrangement. It was tracked down that a cyclic policy could altogether decrease the all-out working time for mixtures with a low measure of light key components. But, more clear rules for different mixtures were hard to form, because the investigation relies upon the mixture, of the column number of trays and the purity of distillate and recovery specifications.

Peng et al. [10] applied Sørensen's system focusing on the reflux drum hold-up and the plate hold-up dynamics during the operating time. The researcher showed by simulation and experiments what these hold-up dynamics affecting on optimal product composition for a given number of cycles. Prior, Sørensen and Skogestad [11] proposed an optimal reflux strategy dependent on cycles and saw that the number of cycles increases as the batch time decline.

Stojkovic et al. [12] examined the distillate optimal control issue of $\mathrm{BD}$ for the non-ideal binary zeotropic systems. The describe equation depends on full column dynamics and manipulating the distillate flow rate to be used as a control variable rather than the reflux, where the purity of distillate composition is taken as another state variable.

Noda et al. [13] examined the optimal working strategy, which limits the energy utilization in complete reflux neglecting both hold-up dynamics and constant vapor flow rate. The researcher showed that the maximum performance was accomplished by optimizing the reflux flow rate, subsequently changing the reboiler and reflux drum hold-ups with time. In a composition control, most early researchers studied the implementation of a controller incorporated with an assortment of a state estimator in which compositions in all column plates were measured.

In 1998, Barolo and Berto [14] proposed a control technique dependent on modified Nonlinear Internal Model Control (NIMC) structure combined with an Extended Luenberger Observer (ELO) is utilized for nonlinear Single-Input Single-Output (SISO) systems. The state transformation relation with the nonlinear observer sanctioned structure was created by Zeitz, in 1987 [15].

It can be using the eigenvalue operator to dimension a nonlinear observer without solving the nonlinear partial differential equations for the transformation if the transformed nonlinearities are linearized about the reconstructed state.
Concerning the extended Kalman Filter (KF) algorithm, this nonlinear observer configuration is known as Extended Luenberger Observer (ELO) for control to achieve a constant concentration of the distillate for the BD. Also showed a benefit of the methodology using a regular proportional plus integral controller. In any case, they detailed that the ELO exactness was likely to degrade if the tray hydraulic was taken into account, and the observer was considered. The utilization of a stochastic estimator like a Kalman Filter (KF) was suggested when a lot of noise was expected [4].

Li et al. [16] considered the ordinary BD and center vessel BD by modified variable and constant reflux operations in the traditional BD. For two control strategies one of which was mentioned the composition control strategy with an adjusted level-set point, and the other was mentioned the flowrate-restricting control strategy. Subsequently, the center vessel BD had the option at the same time to separate the parts of the ternary mixture. Contrasting the two control strategies showed that the flowrate-restricting control strategy was superior to the composition control strategy with an adjusted level-set point regarding their applicability. Also, Li et al. in 2019 [17] designed double column batch column to separate ternary azeotropes system "tetrahydrofuran, methanol, water" to separate such distillate mixtures based on the residue curve maps.

All above observations show the control distillate with a constant composition is very challenging even for a binary mixture system due to continuous changes over time results in high nonlinearity and non-stationary for distillate product $x_{D}$. The present study, we suggest to adopted relation for the reflux ratio at specific time related within bottom concentration in the pot $x_{B}$ based on material balance, modified by using a new control methodology considering $\mathrm{BD}$ dynamics time. The dynamic time is the sum of the time for vapor flow toward the top tray and the time for condensed liquid flow gotten back from the top tray to the boiler (pot). The modified relation considers the delay time to anticipate quick and stabilize the response of overhead concentration despite the decreasing bottom concentration with time. To calculate this time, we use electroconductive tracer $(\mathrm{KCl})$ injected the bottom pot and the top tray.

The modified relation implemented with controller timing loop using LabView on BD column by manipulating solenoid valve (controls the reflux rate) gives pre timing during the operation to maintain control of the distillate composition with quick response. And overcome the delay time that occurs for the relation involves both compositions of the reboiler and reflux rate to keep constant distillate composition along operating time. A modified relation with dynamic time control gives a good result, as compared without adding the dynamic as pre-time $(+d t)$.

\section{Theoretical work}

The steps involved in the calculation procedure keep the product concentration constant with Variable Reflux Mode for a binary system. We suggest the following equations from Bauerle and Sandall [18] relying upon the mathemat- 
ical model of the reflux strategy need to use as a control variable $(R)$.

Overall mole balance for the BD system in equation (1):

$$
\mathrm{d} B=-\mathrm{d} D .
$$

A material balance for the light component

$$
\mathrm{d}\left(x_{B} B\right)=-x_{D} \mathrm{~d} D .
$$

Combining equations (1) and (2) results in

$$
\mathrm{d} B / B=\left(\mathrm{d} x_{B}\right) /\left(x_{D}-x_{B}\right) .
$$

Equation (3) may be integrated between the initial charge conditions, $x_{B_{1}}$ and $B_{1}$ and some later conditions, $x_{B_{2}}$ and $B_{2}$.

$$
\mathrm{d} B / B=\left(\mathrm{d} x_{B}\right) /\left(x_{D}-x_{B}\right) .
$$

Equation (4) relates the total moles in the still at any time, $B_{2}$, to the mole fraction of the light component in the still (boiler), $x_{B_{2}}$. This is the basic design equation that describes the separation that the column can achieve. For the constant distillate composition mode of operation, $x_{D}$ is constant and equation (4) can readily be integrated.

A differential balance around the condenser leads to

$$
\mathrm{d} V=\mathrm{d} L+\mathrm{d} D
$$

rearranged

$$
\mathrm{d} V=\mathrm{d} D /((1-\mathrm{d} L / \mathrm{d} V)),
$$

where $\mathrm{d} L / \mathrm{d} V$ is the instantaneous slope of the operating line Figure 1, which may be related to the external reflux ratio.

A differential balance around the condenser leads to

$$
\mathrm{d} L / \mathrm{d} V=R /((R+1)) .
$$

For the constant distillate composition mode of operation, the material balance relation, equation (4), may be integrated directly to give:

$$
\begin{gathered}
\frac{B_{2}}{B_{1}}=\frac{\left(x_{D}-x_{B 1}\right)}{\left(x_{D}-x_{B 2}\right)}, \\
V=\int_{0}^{v} f(x) \mathrm{d} V \\
=B_{1}\left(x_{D}-x_{B_{1}}\right) \int_{x_{w_{1}}}^{x_{w_{2}}} \frac{\mathrm{d} x_{B}}{\left(\left(x_{D}-x_{B}\right)^{2}(1-\mathrm{d} L / \mathrm{d} V)\right)} .
\end{gathered}
$$

To integrate equation (9) for the vapor requirement it is necessary to relate the instantaneous slope of the operating line, $\mathrm{d} L / \mathrm{d} V$, to the mole fraction in the still, $x_{B}$.

Substitution of relative volatility results in

$$
\frac{\mathrm{d} L}{\mathrm{~d} V}=\frac{\left(x_{D}-x_{D} x_{B}(\alpha-1)-\alpha x_{B}\right)}{\left(x_{D}-x_{B}\right)\left(1+x_{B}(\alpha-1)\right)} .
$$

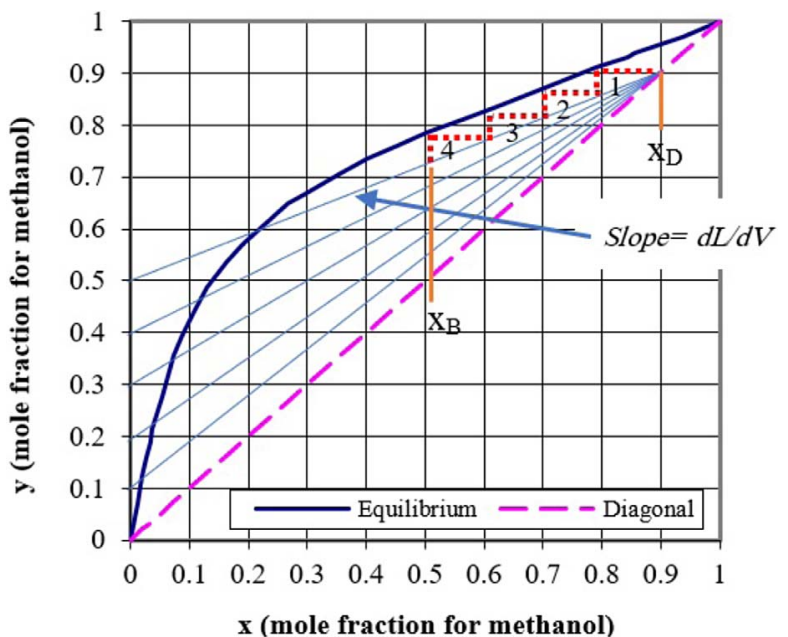

Fig. 1. Vapor-liquid equilibrium for a methanol-water system with the adapted operating line at constant concentration 0.9 .

Solve equation (7) and equation (10) gives equation (11) which represent reflux effect on top and bottom concentrations with the time of the separation process. The instantaneous value of $x_{D}$ as a function of $x_{B}$ [18]:

$$
x_{D}=\frac{\left[(R+1) \alpha x_{B}-R x_{B}-R x_{B}^{2}(\alpha-1)\right]}{\left[1+x_{B}(\alpha-1)\right]} .
$$

In solving equation (11) for average relative volatility $\alpha$ (methanol-water 4.02) and a constant distillate composition $\left(x_{D}=0.9\right)$ to find the relationship of instantaneous value $x_{B}$ as a function reflux ratio for a constant distillate composition mode operation as shown in Table 1.

Table 1. Value of the reflux ratio at a specific time that keeps the distillate concentration constant at 0.90 .

\begin{tabular}{lc}
\hline Methanol conc. in the pot $\left(x_{B}\right)$ & Reflux ratio \\
\hline 0.5 & 0.333 \\
0.45 & 0.424 \\
0.4 & 0.53 \\
0.35 & 0.662 \\
0.3 & 0.81 \\
0.25 & 1.022 \\
0.2 & 1.33 \\
0.18 & 1.5 \\
0.16 & 1.716 \\
0.14 & 2 \\
0.12 & 2.35 \\
0.1 & 2.85 \\
0.08 & 3.6 \\
0.07 & 4.14 \\
0.06 & 4.86 \\
0.05 & 5.86 \\
0.04 & 7.36 \\
\hline
\end{tabular}




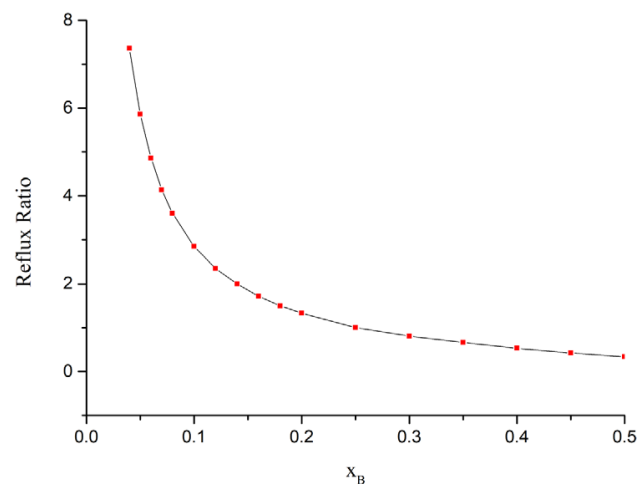

Fig. 2. Theoretical minimum reflux ratios vs. still pot concentration $x_{B}$ are required for a constant distillate composition of $90 \%$ methanol.

Making correlation $x_{B}$ with $R$ from data taken for each case using the following procedure draw the equilibrium line for the system (methanol-water) and locating the desire concentration of distillate (dome concentration).

For a BD, an increase of the reflux ratio $R$ is to accomplish the desired distillate quality. So, it can obtain the relationship between the instant reflux ratio $R$ and the corresponding composition of the remaining liquid in the pot $x_{B}$ keeping $X_{D}$ constant. Figure 2 shows the results from mathematical simulations concerning the present study relation of $R$ with $x_{B}$ give a regression equation (12).

$$
R=0.1837-1.178 x_{B} .
$$

\section{Calculation and applying the mathematical model}

To derive the relation between reflux ratio $R$ and boiler composition $x_{B}$, use McCabe-Thiele graphical method is shown in Figure 1. Several values of $(R)$ were selected, operating lines were drawn through the fixed point at distillate composition $\left(x_{D}, x_{D}\right)$ with slope $\mathrm{d} L / \mathrm{d} V=(R / R+1)$, and steps were drawn between the operating line and the equilibrium curve to obtain the bottom composition $x_{B}$. This recursive scheme is repeated until the desired stopping criterion is met, thus $x_{B}$ can be found at each value of the reflux ratio $R$.

While $R(L / D)$ was changed as shown in Figure 1, the slope of the operating line keeps changing with the fixing point of the intersect $x_{D}$. Solving for a constant number of trays to find the relation of $x_{B}$ with $R$ to keep $x_{D}$ constant, to make this process worked it should be taken by control through manipulating reflux ratio $R$ at a specific time. The correlation of composition $\left(x_{B}\right)$ with $R$ from data taken for each case using the following procedure:

1. Draw the equilibrium line for the system (methanolwater).

2. Locating the desire concentration of distillate composition (dome conc.) in our case we take constant distillate composition at $90 \%$ of methanol.
3. At this point, the expected bottom concentration $x_{B}$ could be determined by fitting 8 trays between the operating line and the equilibrium line.

4. Meanwhile finding the minimum reflux $R_{\min }$ $\left(R=2.0 R_{\min }\right)$ required to achieve the distillate composition as shown in Figure 1.

The proposed correlation of $x_{B}$ and $R$ considering the calculated dynamic time $+d t$ to increase the response for $x_{D}$ at the desired constant mode. To build a typical predictive control for a distillation column for a fast, stable, and constant distillate composition technique is by finding the time required to make changes in the reflux rate to anticipate the concentration drop in the bottom vessel (pot).

In other words, when reading the $x_{B}$ the controller must change $R$ to keep the system run with a constant $x_{D}$. Therefore, there is a time required to make it happen for the response of change to make it happen for the vapor to rise from the bottom vessel through the trays to the final destination in the condenser $\left(t_{1}\right)$. Also, the downtime required to return back to the vessel by the reflux flow $\left(t_{2}\right)$.

These periods $\left(t_{1}, t_{2}\right)$ were estimated by using a tracer dose of $\mathrm{KCl}$ solution $(10 \mathrm{gm} / \mathrm{L})$ that was injected both into the bottom pot and the top tray with reflux. The tracer distribution across the column was monitored by conductivity measurement. Taken time for the column used in this study was $t_{1} 7.21 \mathrm{~min}$ and for $t_{2} 5.4 \mathrm{~min}$ as shown in Figures 3a and $3 \mathrm{~b}$, the total time $t_{1}+\mathrm{t}_{2}$ represents the dynamic time $(+d t)$ that is considered to predict the varying in reflux ratio based on the composition of pot at the specific control time.

From all above, a mathematical model based on mole balance and equilibrium vapor-liquid equation has been developed for $x_{B}$ with $R$ to overcome the delay time for a change to take place as a model to predict action map that used in LabView to achieve the goal that runs the BD at $x_{D}$ in a constant mode.

\section{Experimental works}

The distillation column was in $5 \mathrm{~cm}$ diameter with 8 sieve trays and $1 \mathrm{~m}$ long as shown in Figure 4 . The boil-up rate was kept constant by controlling the heating mantle surrounding the boiler (still pot) with a heat duty of $1000 \mathrm{~W}$. The column works at atmospheric pressure, where the reflux ratio was controlled by a solenoid valve at the top. The system mixture was methanol-water, initial composition $50 \%$ mole of methanol and $50 \%$ mole of water in all experiments runs. Temperature sensors (thermocouples) were placed at each distillation tray.

The boiler (still pot) was charged and heated up under total reflux until the steady-state conditions were achieved. After, the reflux ratio $R$ was turned from infinity to the finite reflux ratio, and the product was withdrawn at the top of the column.

During the column operations, the distillate flowrate profile, the concentration profiles of the liquid phase along the column, and the temperature profiles were measured for all trays, pot, and condenser was interfaced to the 

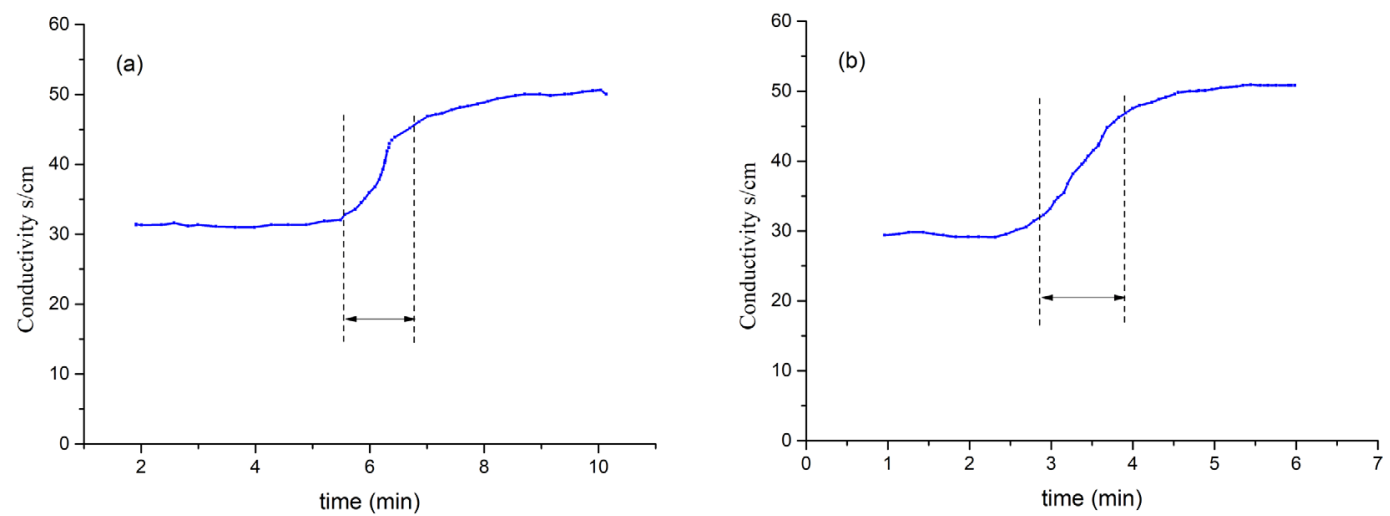

Fig. 3. (a) The response time required for the tracer injected into the pot to achieve the distillate accumulator. (b) The required time for the tracer that injected in the distillate accumulator to achieve the still pot.

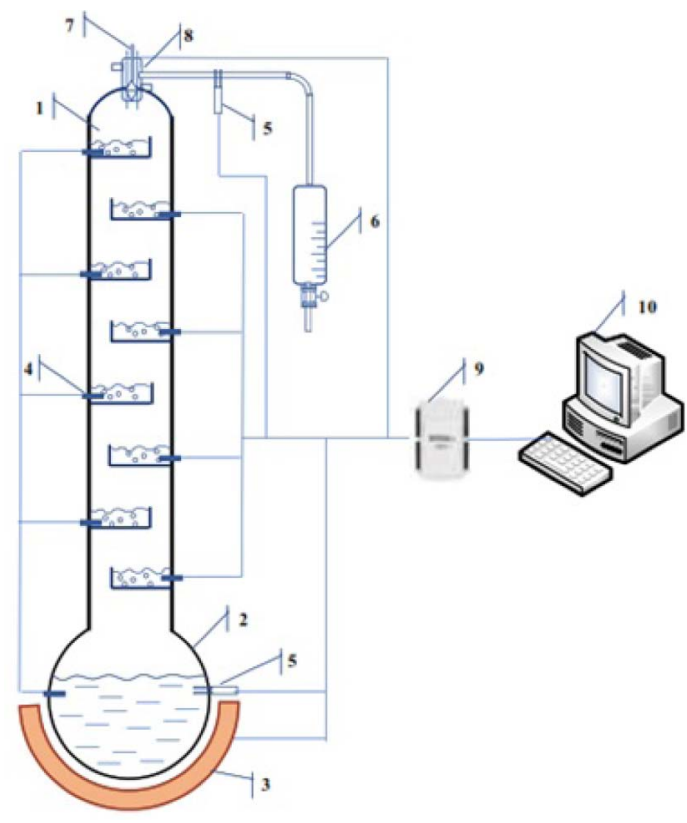

Fig. 4. Schematic diagram for the batch distillation column $(1-$ Vessel; 2 - Still Pot (boiler); 3 - Heating mantle; 4 Temperature sensors; 5 - Condenser; 6 - Product receiver; 7 Solenoid valve; 8 - Conductivity; 9 - DAQ; 10 - PC.

computer. The computer interface used was NI USB-6211 data acquisition card which can support 16 Analog inputs for temperature sensors and 2 Analog output channels that's control the heat rate of heating mental and a Solenoid valve was used to control the reflux rate. The complete experimental controlled distillation column is shown in Figure 4.

\section{Results and discussion}

A model equation has been written with LabVIEW to run the whole process of batch distillation column at constant distillate composition mode due to its equilibrium at a

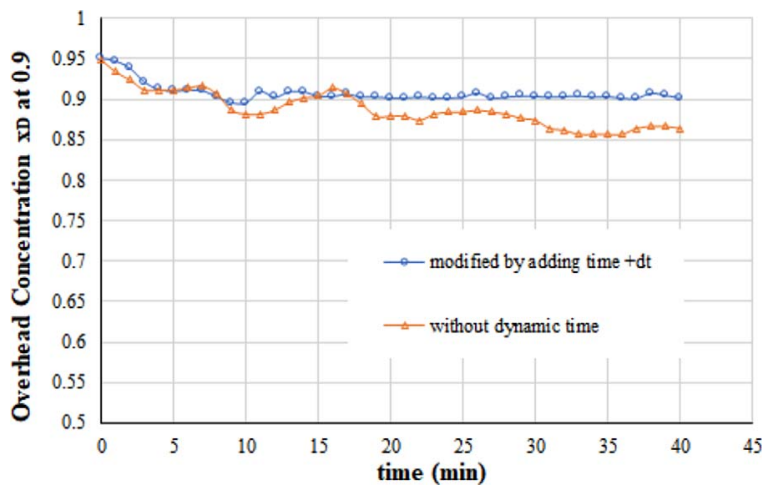

Fig. 5. Overhead concentration at setup points at 0.9 with/ without adding dynamic-time $(+d t)$.

specific point, these are input as a data signal from temperature sensors where the output signal controls the solenoid valve that involves changes for reflux rate.

A dynamic model based on equilibrium stages correlations implemented in LabVieu ${ }^{\circledR}$ and validated for a multi-tray column operating under a varying reflux ratio to separate a methanol/water mixture.

Figure 5 shows applying the protocol described above as pre-time determined using the tracer method to anticipate the delay in the process due dynamic time in the LabView ${ }^{\circledR}$, the model agreed with the simple equation (14). Considering the case of methanol-water in a batch column with $N=8$ plates, the pre-time in control loop time policy was compared with instantaneous time equation (13) and increasing purity as step change of the distillate composition as a final product:

$$
\begin{gathered}
R(t)=f\left(x_{B}(t)\right), \\
R(+\mathrm{d} t)=f\left(x_{B}(t)\right) .
\end{gathered}
$$

On the other hand, when taking into account equation (14) and working on it within the control program, we notice the stability of the response. Furthermore, we can believe that 


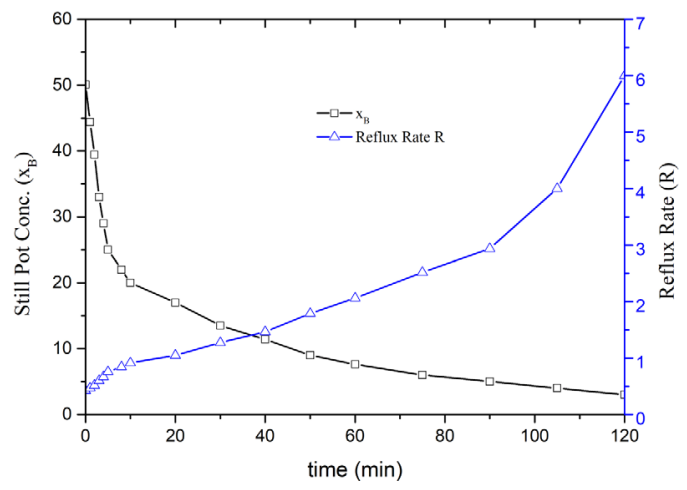

Fig. 6. Bottom concentration vs. variable reflux that maintains constant distillate concentration at 0.90 .

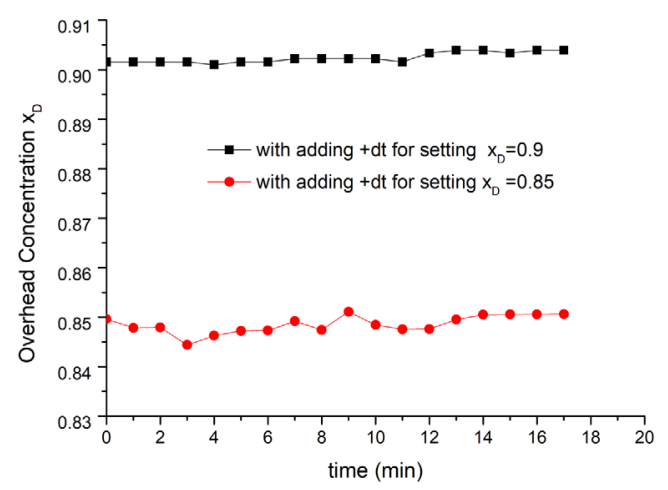

Fig. 7. Overhead concentration for setpoints at 0.85 , and 0.9 with dynamic-time $(+d t)$.

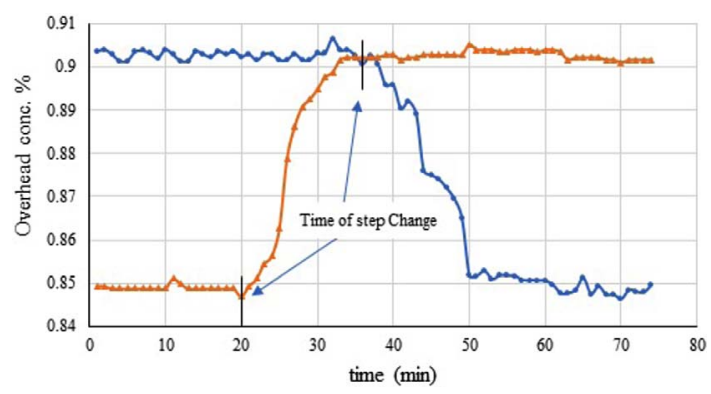

Fig. 8. Overhead concentration disturbance from 0.85 to 0.9 with dynamic-time $(+d t)$.

the light component $x_{D}$ more stable along the operation time, and the production phase is fast and smooth in the case of adding dynamic time, and the composition control is tight until the end of the operation. Figure 6 shows the real-time relationship between the $x_{B}$ reading and the amount of $R$ needed to control the constant distillate composition mode as an output value.
To check the Validation for the suggested model and its stability and the operability of the batch distillation, we disturb the system by changing the setpoint of the distillate composition as an example from 0.85 to 0.9 and vice versa to the system checking reading the response for $x_{D}$ as shown in Figure 7.

The control policy for the reflux flow rate (Fig. 8) is approximated as a succession of reflux at time $t$ with reflux at time $+d t$ with the same duration. Compare both software in terms of control variable reflux and constant distillate composition response for Batch Column.

\section{Conclusion}

Literature works noticed that complexity controlling distillate composition in batch distillation control problem. In this work, the proposed batch column separates a binary mixture (methanol-water) in two different methods to control the distillate composition by varying reflux rate based on the boiler concentration. First is the instant time control of the distillate with varying reflux rates. The second method is modified by considering the time delay due to the dynamic behaviour. Time for both vapor upstream through the trays and refluxed liquid downstream through the trays to the bottom column using an electroconductive tracer. However, the mathematic relation for the reflux rate with bottom concentration was implemented easily using LabVIEW software to control the operation. The corresponding experiments confirm that the improved reflux policy column (adding the predicted time) is practicable and easy to implement, operate, and of great practical importance in controlling distillate products. Reduces the separation time required for the distillation process, thus reducing the operating energy consumed. Finally, we believe that further research in this field should address the problem of selecting the best method to measure the dynamic time required for the $R$ changes to keep $x_{D}$ constant, when the system is complicated. In the case of separation multicomponent mixtures, the proposed control strategy can be easily extended to control the desired distillate purity. The dynamic time delay for the batch column used in this study was about 12 min was estimated by adding a conductive tracer both to the boiler and the reflux accumulator. Add the dynamic time delay within the equation relations of reflux ratio and boiler concentration as predicting the change in reflux rate policy to obtain the desired constant distillate composition. To overcome the delay problem for a response and keep distillate product with the desired purity using pre-time is more performant for variable reflux $R[+d t]$ was prompted to get a quick response for the desired constant $x_{D}$. This leads to distillate concentration stabilized by manipulating the reflux rate. For example, keep $x_{D}=0.9$ when working with the controlling program using equation (14) it can notice the loss of control after a period due to accumulated errors as a result of the delay time when sensing to the concentration of $x_{B}$ and the change of the value of reflux responded to get a fixed $x_{D}$. Manipulating the reflux without taking effect of the delayed time, leads to accumulating error on the 
response caused a loss in the controlled overhead product $x_{D}$. The results are confirmed by disturbing the control system of the batch distillation, showing that the improved reflux policy by 13-15 min ahead to get the desired overhead product $x_{D}$. Finally, we believe that further research in this field should address the problem of selecting the best method to measure the dynamic time required for the $R$ changes to keep $x_{D}$ constant.

\section{References}

1 Kockmann N. (2014) History of distillation, in: Distillation, Academic Press, pp. 1-43.

2 Gruetzmann S., Kapala T., Fieg G. (2006) Dynamic modelling of complex batch distillation starting from ambient conditions, Comput. Aided Chem. Eng. 21, 865-870.

3 Garcia A.N., Loria J.C.Z., Marin A.R., Quiroz A.V.C. (2014) Simple multicomponent batch distillation procedure with a variable reflux policy, Braz. J. Chem. Eng. 31, 2, 531-542.

4 Weerachaipichasgul W., Kittisupakorn P., Saengchan A., Konakom K., Mujtaba I.M. (2010) Batch distillation control improvement by novel model predictive control, J. Ind. Eng. Chem. 16, 2, 305-313.

5 Galindez H., Fredenslund A.A. (1988) Simulation of multicomponent batch distillation processes, Comput. Chem. Eng. 12, 4, 281-288.

6 Diwekar U.M., Madhavan K.P. (1991) Batch-Dist: A comprehensive package for simulation, design, optimization and optimal control of multicomponent, multifraction batch distillation columns, Comput. Chem. Eng. 15, 12, 833-842.

7 Furlonge H.I. (2000) Optimal operation of unconventional batch distillation columns.
8 Bogart M.J.P. (1937) The design of equipment for fractional batch distillation, Trans. Am. Inst. Chem. Eng. 33, 139.

9 Sørensen E. (1999) A cyclic operating policy for batch distillation-theory and practice, Comput. Chem. Eng. 23, $4-5,533-542$.

10 Peng B., Shuang S., Sheng M., Xiaofeng L.I. (2010) A dynamic modeling for cyclic total reflux batch distillation, Chin. J. Chem. Eng. 18, 4, 554-561.

11 Sørensen E., Skogestad S. (1994) Optimal operating policies of batch distillation with emphasis on the cyclic operating policy, in: Proceedings of the 5th International symposium on Process Systems Engineering (PSE'94), pp. 449-456.

12 Stojkovic M., Gerbaud V., Shcherbakova N. (2018) Cyclic operation as optimal control reflux policy of binary mixture batch distillation, Comput. Chem. Eng. 108, 98-111.

13 Noda M., Kato A., Chida T., Hasebe S., Hashimoto I. (2001) Optimal structure and on-line optimal operation of batch distillation column, Comput. Chem. Eng. 25, 1, 109-117.

14 Barolo M., Berto F. (1998) An advanced strategy for composition control in batch distillation, IFAC Proceedings Volumes 31, 11, 429-434.

15 Zeitz M. (1987) The extended Luenberger observer for nonlinear systems, Syst. Cont. Lett. 9, 2, 149-156.

16 Li X., Zhao T., Wang Y., Wang Y., Zhu Z. (2018) Operational design and improvement of conventional batch distillation and middle-vessel batch distillation, Braz. J. Chem. Eng. 35, 2, 769-784.

17 Li X., Yang X., Wang S., Yang J., Wang L., Zhu Z., Gao J. (2019) Separation of ternary mixture with double azeotropic system by a pressure-swing batch distillation integrated with quasi-continuous process, Proc. Saf. Environ. Prot. 128, 85-94.

18 Bauerle G.L., Sandall O.C. (1987) Batch distillation of binary mixtures at minimum reflux, AIChE J. 33, 6, 10341036. 